\title{
Vascular function assessed with cardiovascular magnetic resonance predicts survival in patients with advanced chronic kidney disease Patrick B Mark*1,2, Arthur Doyle ${ }^{2}$, Kevin G Blyth ${ }^{3}$, Rajan K Patel ${ }^{1,2}$, Robin AP Weir ${ }^{3}$, Tracey Steedman ${ }^{3}$, John E Foster ${ }^{3}$, Henry J Dargie ${ }^{3}$ and Alan G Jardine ${ }^{1,2}$
}

Address: ${ }^{B}$ BHF Glasgow cardiovascular research centre, faculty of medicine, University of Glasgow, Glasgow, Scotland, G12 8TA, UK, ${ }^{2}$ Renal unit, Western infirmary, Glasgow, Scotland, G11 6NT, UK and ${ }^{3}$ Department of cardiology, Western infirmary, Glasgow, Scotland, G11 6NT, UK

Email: Patrick B Mark* - p.mark@clinmed.gla.ac.uk; Arthur Doyle - arthur.doyle@gmail.com; Kevin G Blyth - kblyth@btinternet.com; Rajan K Patel - rkp4b@clinmed.gla.ac.uk; Robin AP Weir - robinweir75@hotmail.com; Tracey Steedman - traceysteeds@hotmail.com; John E Foster - j.e.foster@clinmed.gla.ac.uk; Henry J Dargie - h.dargie@bio.gla.ac.uk; Alan G Jardine - A.Jardine@clinmed.gla.ac.uk

* Corresponding author

Published: 18 August 2008

Journal of Cardiovascular Magnetic Resonance 2008, 10:39 doi:10.1186/1532-429X-10-39

This article is available from: http://www.jcmr-online.com/content/10/1/39

(c) 2008 Mark et al; licensee BioMed Central Ltd.

This is an Open Access article distributed under the terms of the Creative Commons Attribution License (http://creativecommons.org/licenses/by/2.0), which permits unrestricted use, distribution, and reproduction in any medium, provided the original work is properly cited.
Received: 28 April 2008

Accepted: 18 August 2008

\begin{abstract}
Background: Increased arterial stiffness is associated with mortality in patients with chronic kidney disease. Cardiovascular magnetic resonance (CMR) permits assessment of the central arteries to measure aortic function.

Methods: We studied the relationship between central haemodynamics and outcome using CMR in 144 chronic kidney disease patients with estimated glomerular filtration rate $<15 \mathrm{ml} / \mathrm{min}$ (I I0 on dialysis). Aortic distensibilty and volumetric arterial strain were calculated from cross sectional aortic volume and pulse pressure measured during the scan.

Results: Median follow up after the scan was 24 months. There were no significant differences in aortic distensibilty or aortic volumetric arterial strain between pre-dialysis and dialysis patients. Aortic distensibilty and volumetric arterial strain negatively correlated with age. Aortic distensibilty and volumetric arterial strain were lower in diabetics, patients with ischaemic heart disease and peripheral vascular disease. During follow up there were 20 deaths. Patients who died had lower aortic distensibilty than survivors. In a survival analysis, diabetes, systolic blood pressure and aortic distensibilty were independent predictors of mortality. There were 12 non-fatal cardiovascular events during follow up. Analysing the combined end point of death or a vascular event, diabetes, aortic distensibilty and volumetric arterial strain were predictors of events.
\end{abstract}

Conclusion: Deranged vascular function measured with CMR correlates with cardiovascular risk factors and predicts outcome. CMR measures of vascular function are potential targets for interventions to reduce cardiovascular risk.

\section{Introduction}

Premature cardiovascular disease is the leading cause of death in patients with end stage renal disease (ESRD) treated with dialysis. The relationship between cardiovascular (CV) risk factors and CV events is less clear in ESRD than in the general population, with paradoxical relation- 
ships between both cholesterol and blood pressure and $\mathrm{CV}$ risk[1,2]. The presence of increased arterial stiffness is a predictor of CV morbidity and mortality in $\operatorname{ESRD}[3,4]$. Conventionally, this has been assessed by measurement of pulse wave velocity (PWV). More recently, cardiovascular magnetic resonance (CMR) has been used to identify tissue abnormalities in the heart of ESRD patients [5]. This imaging modality also permits contemporaneous visualisation of large arteries and direct measurement of aortic function, providing an integrated assessment of both ventricular and vascular function in one examination. Aortic distensibilty $(\mathrm{AD})$, the relative change in aortic size throughout the cardiac cycle relative to blood pressure, is readily assessed with CMR. Given the strong relationship between vascular stiffness and outcome in ESRD, CMR measures of vascular function may identify patients at increased CV risk. We have demonstrated in a previous pilot study using CMR that aortic stiffness is increased in patients with ESRD[6]. More recently aortic stiffness has been demonstrated with CMR to be increased in patients with even mild chronic kidney disease (CKD), equivalent to findings in patients with heart failure with preserved systolic function[7].

To date however, the relationship between aortic distensibilty (AD), as measured with CMR and long term outcome has not been assessed. Thus, we assessed the relationship between CMR measures of vascular function and conventional $\mathrm{CV}$ risk factors in ESRD patients and studied the long term prognostic implications of CMR measures of vascular function.

\section{Methods \\ Subjects}

Studies were performed on 144 patients with chronic kidney disease (CKD) stage 5[8]. All patients were assessed as part of a CV screening program for renal transplantation and therefore were either on dialysis therapy or expected to start dialysis within six months. All patients gave written, informed consent and the study was approved by the local ethics committee.

\section{CMR technique}

CMR was performed using a 1.5 Tesla MR scanner (Sonata, Siemens, Erlangen, Germany). In haemodialysis patients imaging was performed on the post-dialysis day whereas peritoneal dialysis patients were studied at their "dry weight". Aortic volume was acquired from cine CMR images in the transverse plane of the ascending aorta, obtained at the level of the main pulmonary artery, using a steady-state free precession (true FISP) sequence, TR = $3.2 \mathrm{~ms}, \mathrm{TE}=1.6 \mathrm{~ms}, \mathrm{FA}=60^{\circ}$, FoV $276 \times 340 \mathrm{~mm}$, pixel dimensions $2.3 \times 1.3 \mathrm{~mm}$, slice thickness $=7 \mathrm{~mm}$ (Figure 1). The approximately 10 second breath-hold CMR resulted in images with a temporal resolution of $22.5 \mathrm{~ms}$.
During $\mathrm{AD}$ measurement brachial blood pressure was measured using an oscillometric device (Schiller Magscreen, Schiller AG, Baar, Switzerland).

Left ventricular (LV) mass and function were measured using a true FISP sequence to acquire cine images in long axis planes (vertical long axis, horizontal long axis, LV outflow tract) followed by sequential short axis LV cine loops ( $8 \mathrm{~mm}$ slice thickness, $2 \mathrm{~mm}$ gap between slices) from the atrio-ventricular ring to the apex. LV mass index (LVMI) was calculated by normalising LV mass to the body surface area. Overall, scan time was approximately 30 minutes.

All CMR analyses were performed using conventional analysis software (Argus, Siemens, Erlangen, Germany). Cross sectional aortic contours were defined by manual planimetry throughout the cardiac cycle, with resulting aortic volume defined as the measured cross sectional area multiplied by the slice thickness. This calculation was performed automatically by the analysis program.

\section{Measurement of aortic distensibilty and volumetric arterial strain}

Aortic distensibilty (AD) was calculated from change in aortic volume and simultaneous brachial blood pressure using the formula:

Aortic distensibilty $=\frac{\left[(\text { Aortic volume })_{\max }-(\text { Aortic volume })_{\min }\right]}{\left[(\text { Aortic volume })_{\min } * \text { pulse pressure }\right]}$

where (Aortic volume) $)_{\max }$ and (Aortic volume) min $_{\text {are }}$ are maximal and minimal calculated aortic volumes during the cardiac cycle. Additionally, aortic volumetric arterial strain (VAS), which is non-pressure dependent, was calculated from the formula:

$$
\mathrm{VAS}=\frac{\left[(\text { Aortic volume })_{\max }-(\text { Aortic volume })_{\min }\right]}{\left[(\text { Aortic volume })_{\min }\right]}
$$

As a ratio of change in aortic volume, VAS does not have any units.

\section{Clinical data}

All patients underwent conventional cardiovascular risk factor assessment including history, clinical examination, ECG as well as routine haematology, biochemical, and lipid profile. A history of ischaemic heart disease (IHD) was defined as previous clinically documented myocardial infarction/angina pectoris or previous coronary revascularisation procedure. Peripheral vascular disease was defined by clinically documented intermittent claudication with prior consultation by a vascular surgeon or the need for a previous peripheral vessel revascularisation procedure. Blood was drawn at the time of scanning for 

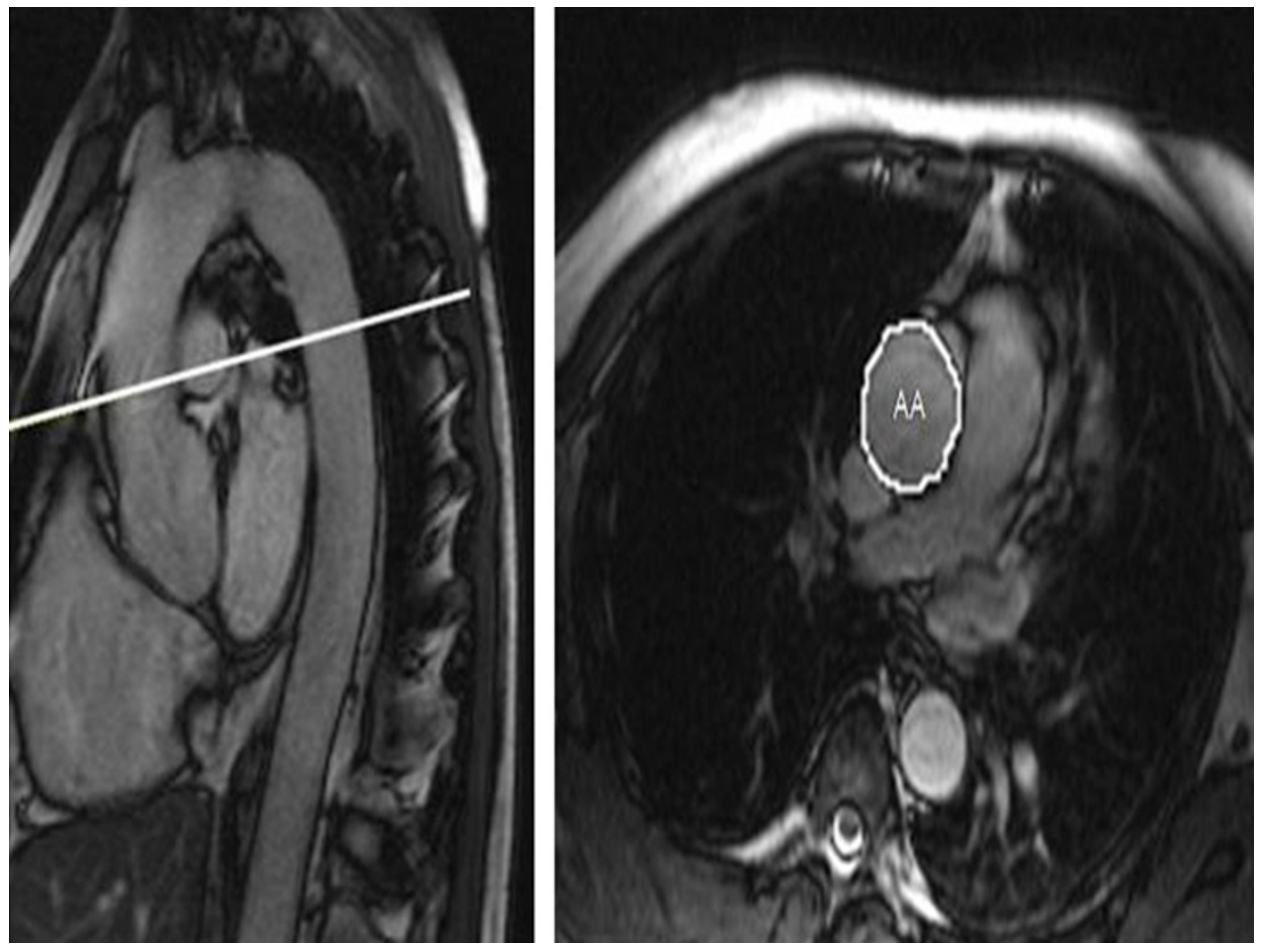

Figure I

Representative images demonstrating sagittal view (left) used to plan transverse plane aortic images (right) with schematic tracing of ascending aorta (AA).

lipids and $\mathrm{C}$ - reactive protein, with other haematological and biochemical parameters (haemoglobin, dialysis adequacy, calcium-phosphate product) assessed from the mean of readings taken on the three consecutive months preceding the scan.

\section{Follow up}

Follow up data was collected from date of the CMR scan using electronic patient records. Death and cardiovascular events (myocardial infarction, cerebrovascular event, coronary revascularisation and amputation for peripheral vascular disease) were collected as end points.

\section{Statistical methods}

Correlations between cardiac dimensions, measures of arterial function and continuous clinical variables were assessed with Pearson and Spearman co-efficients. Differences between groups were tested by student's t-test and Mann-Whitney-U test. Measures of vascular function were compared between patients who died or had a vascular event during the follow up period with these measures divided into tertiles or quartiles and subjected to an unadjusted survival analysis by the Kaplan-Meier method. Statistical significance was determined by the log-rank test. Cox survival analysis was performed to assess the influence of multiple variables on outcome. Variables identi- fied as possibly influential on outcome by univariate analysis were then entered into a forward stepwise regression model. As exponential values logarithmic transformation was used for $\mathrm{AD}$ and VAS values to simplify interpretation of values in the regression analyses. Analyses were performed using SPSS 13.0 software package (SPSS Inc., Chicago, IL., USA).

\section{Results}

Subjects

Patient demographics at the time of scan are shown in Table 1. 144 patients had aortic VAS measurement and 122 patients had measurement of AD available for analysis. 22 patients did not have blood pressure readings to calculate AD (either due to bilateral arteriovenous fistulae used as vascular access for dialysis making recording impossible, or due to blood pressure being recorded nonsynchronously with the AD trace).

\section{Clinical correlates of aortic distensibilty and volumetric arterial strain}

All scans were analysed by a single observer (P.B.M). No significant differences in patient demographics or vascular function were exhibited between patients with advanced renal failure not yet on dialysis therapy (34 patients; $24.6 \%$ ) and those established on dialysis (110 patients) 
Table I: Background demographics of patients studied

\begin{tabular}{lll}
\hline Number & 144 & \\
Age & 51.5 & $(11.2)$ \\
Male (\%) & 90 & $(62.5)$ \\
Height (m) & 169.5 & $(9.8)$ \\
Weight (kg) & 75.9 & $(16.1)$ \\
On dialysis (\%) & 110 & $(76.4)$ \\
$\quad$ Haemodialysis & 61 & $(42.4)$ \\
$\quad$ Peritoneal dialysis & 49 & $(34.0)$ \\
RRT time (months) & 6.0 & $(42.0)$ \\
Past history of IHD (\%) & 24 & $(16.7)$ \\
Diabetes (\%) & 46 & $(31.9)$ \\
Smoker (\%) & & \\
$\quad$ Never & 83 & $(57.6)$ \\
$\quad$ Current & 38 & $(26.4)$ \\
$\quad$ Ex & 23 & $(16.0)$ \\
SBP (mmHg) & 140.2 & $(24.2)$ \\
DBP (mmHg) & 82.9 & $(12.8)$ \\
Cross sectional aortic volume $\left(\mathrm{mL}^{-1}\right)$ & 5.0 & $(2.0)$ \\
Aortic distensibilty (x I0-3 $\left.\mathrm{mmHg}^{-1}\right)$ & 2.4 & $(2.0)$ \\
Aortic volumetric arterial strain & 0.13 & $(0.09)$ \\
\end{tabular}

Results are show as mean with standard deviation in parenthesis or number with percentage in parenthesis as appropriate, except for RRT time and measures of vascular function which are displayed as median and inter quartile range.

and therefore patients were combined as a single group. $\mathrm{AD}$ and VAS demonstrated a negative correlation with age (AD R $=-0.44, \mathrm{p}<0.001$, VAS $\mathrm{R}=-0.44, \mathrm{p}<0.001)$.

There were no significant correlations between haemoglobin, dialysis adequacy (urea reduction ratio in haemodialysis or creatinine clearance in peritoneal dialysis), time on dialysis, lipid parameters, C-reactive protein, calcium, phosphate or calcium phosphate product and AD or VAS. There were no significant differences in AD or VAS between genders. AD and VAS were reduced in patients with diabetes mellitus (diabetics - median AD $1.8 \times 10^{-3}$ vs. non-diabetics $2.8 \times 10^{-3} \mathrm{mmHg}^{-1}, \mathrm{p}=0.001$; VAS 0.11 vs. $0.15, \mathrm{p}=0.001)$, patients with a history of ischaemic heart disease (median AD $1.8 \times 10^{-3}$ vs. $2.6 \times 10^{-3} \mathrm{mmHg}^{-}$ $1, \mathrm{p}=0.028$; VAS 0.11 vs. $0.15, \mathrm{p}=0.009$ ) or peripheral vascular disease (median aortic distensibilty $1.2 \times 10^{-3} \mathrm{vs}$. $1.8 \times 10^{-3} \mathrm{mmHg}^{-1}, \mathrm{p}=0.005$; VAS 0.14 vs. $0.07, \mathrm{p}=$ $0.015)$. No significant differences in AD or VAS were demonstrated between patients treated with statins or requiring antihypertensive therapy. There were no significant differences between AD between dialysis modalities. 58 patients $(40.3 \%)$ had a functioning arteriovenous fistula or graft at the time of scanning. There were no significant differences in $\mathrm{AD}$ or VAS between patients with an arteriovenous fistula or graft and those without.

Weak but statistically significant negative correlations were demonstrated between AD and LVMI $(\mathrm{R}=-0.21, \mathrm{p}=$ $0.021)$ and end systolic volume $(\mathrm{R}=-0.18, \mathrm{p}=0.048)$ but no other LV dimension. Aortic VAS correlated with markers of cardiac function - left ventricular ejection fraction $(\mathrm{R}=0.23, \mathrm{p}=0.006)$ and stroke volume $(0.19, \mathrm{p}=0.024)$.

\section{Relationship between CMR measures of vascular function on survival and combined mortality and cardiovascular events}

Follow up data were available for all 144 patients. Overall survival data was analysed for the whole cohort including analyses of the relationship between VAS and outcome. For analyses of the relationship between $\mathrm{AD}$ and outcome, only the 120 patients who only results of AD available were studied. The median follow up period was 719 days (interquartile range 375 days). There were 20 deaths, giving an overall mean death rate of 76.2 per 1000 patient years. Patients who died had significantly lower AD than those who where alive at the end of follow up. There were no significant differences in VAS between survivors and patients who died. Patients who died had significantly higher systolic and pulse pressure than survivors (Figures $2,3)$, but there were no significant differences in diastolic blood pressure (Table 2).

During the follow up period there were an additional 12 non-fatal CV events (five patients had a myocardial infarction; four patients underwent coronary revascularisation, two patients undergoing amputation for peripheral vascular disease and one patient had a cerebrovascular event). These patients had significantly lower AD and VAS than those who remained event free (median AD in patients with CV events $1.5 \times 10-3$ vs. $2.8 \times 10-3 \mathrm{mmHg}-1, \mathrm{p}=$ 0.006 ; VAS 0.12 vs. $0.14, \mathrm{p}=0.012$; Figure 4 ). There were no significant difference in blood pressure between

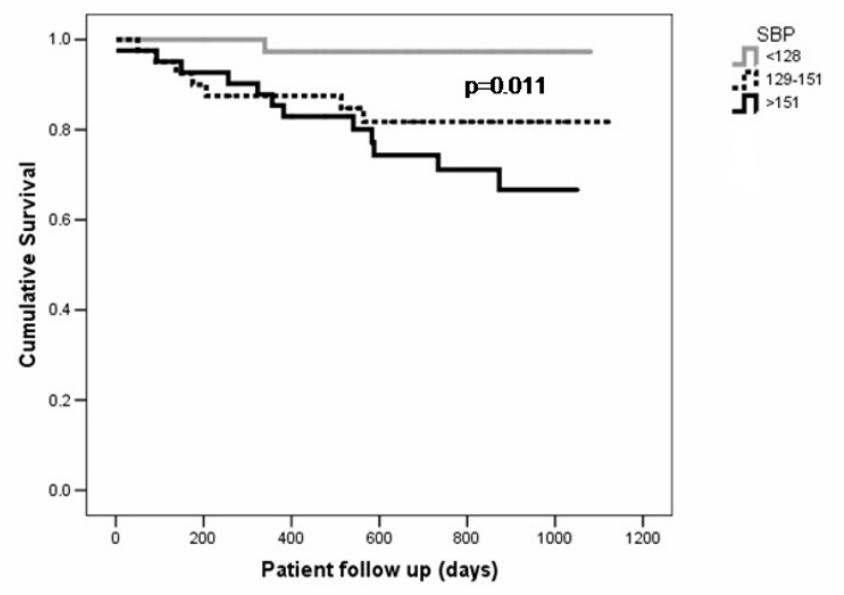

Figure 2

Kaplan-Meier survival curves for all cause mortality with patients stratified by systolic blood pressure tertile. 


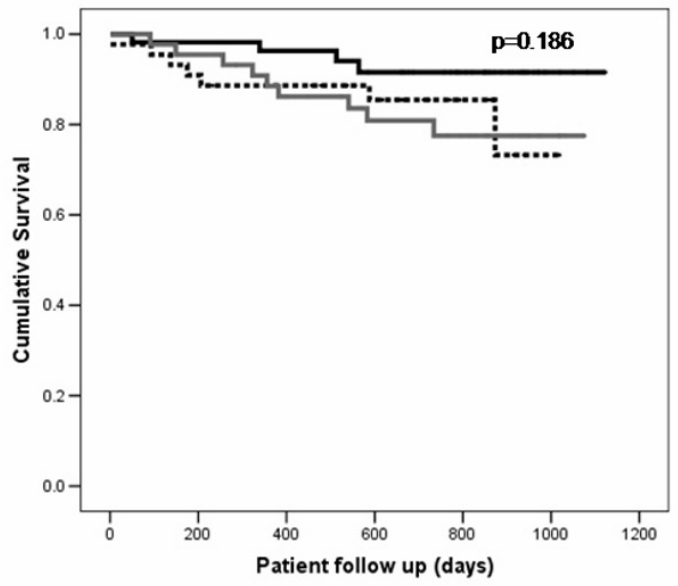

AD tertile $\prod_{\text {High }}$ IT Medium

Figure 3

Kaplan-Meier survival curves for all cause mortality with patients stratified by aortic distensibilty tertile.

patients who remained event free and patients who had a non fatal CV event.

Combining death with non fatal CV events as a combined $\mathrm{CV}$ end point, patients who reached this combined end point had significantly lower AD (median AD $1.7 \times 10^{-3}$ vs. $\left.2.7 \times 10^{-3} \mathrm{mmHg}^{-1}, \mathrm{p}<0.001\right)$, VAS (0.12 vs. $0.14, \mathrm{p}=$ 0.006 ) and higher systolic blood pressure (mean $152.9 \mathrm{vs.}$ $136.5 \mathrm{mmHg}, \mathrm{p}=0.001$ ) and pulse pressure (mean 67.0 vs. $54.5 \mathrm{mmHg}, \mathrm{p}=0.001$ ) than those who did not. There were no significant differences in diastolic blood pressure (mean 85.9 vs. $82.0 \mathrm{mmHg}, \mathrm{p}=0.154$ ) between those who reached the combined end point and those who did not.

In a Cox forward stepwise regression model assessing patient survival; diabetes, systolic blood pressure and $\mathrm{AD}$ were significant independent predictors of patient survival (Table 3). In a similar analysis assessing the combined CV end point of death or a vascular event; diabetes, $\mathrm{AD}$ and aortic VAS were significant predictors of events (Table 4). Due to their close interdependence, blood pressure variables and $\mathrm{AD}$ were entered individually into the each model to determine their influence. Similarly either $\mathrm{AD}$ or VAS was entered individually to the model but not together.

\section{Discussion}

This is the first study to examine the relationship between $\mathrm{AD}$ assessed with CMR and outcome in ESRD. Patients with ESRD have previously been demonstrated to have increased aortic stiffness compared to controls, represented by reduced AD and VAS with associated vessel dilatation[6]. In keeping with the relationship between arteriosclerosis and advancing age, there was a significant relationship between reduced $A D$ and VAS and age. AD and VAS were both lower in patients at highest CV risk, i.e. those with diabetes, ischaemic heart disease and/or peripheral vascular disease. No other factors such as drug therapy, adequacy of dialysis, duration of renal replacement therapy, dialysis modality or any laboratory parameter appeared to be significantly associated with $\mathrm{AD}$ or VAS.

Table 2: Demographic data for patients who were alive and dead at the end of the follow up period

\begin{tabular}{|c|c|c|c|c|c|}
\hline \multirow[b]{2}{*}{ Number } & \multicolumn{2}{|c|}{ Alive } & \multicolumn{2}{|c|}{ Dead } & \multirow[t]{2}{*}{ p value } \\
\hline & 124 & $(86.1)$ & 20 & (13.9) & \\
\hline Age & 51.5 & (II.3) & 51.9 & (II.I) & 0.876 \\
\hline Male (\%) & 80 & $(64.5)$ & 10 & $(50.0)$ & 0.213 \\
\hline On dialysis (\%) & 92 & (74.2) & 18 & $(90.0)$ & 0.122 \\
\hline RRT time (months) & 6.0 & $(26.0)$ & 36.0 & $(107.0)$ & 0.023 \\
\hline Past history of IHD (\%) & 19 & $(15.3)$ & 5 & $(25.0)$ & 0.281 \\
\hline Diabetes (\%) & 34 & (27.4) & 12 & $(60.0)$ & 0.004 \\
\hline \multicolumn{6}{|l|}{ Smoker (\%) } \\
\hline Never & 73 & $(58.9)$ & 10 & $(50.0)$ & \\
\hline Current & 30 & $(24.2)$ & 8 & $(40.0)$ & 0.304 \\
\hline Ex & 21 & $(16.9)$ & 2 & $(10.0)$ & \\
\hline $\mathrm{SBP}(\mathrm{mmHg})$ & 137.3 & (23.6) & 156.3 & $(21.4)$ & 0.001 \\
\hline $\mathrm{DBP}(\mathrm{mmHg})$ & 82.1 & $(12.9)$ & 87.4 & (II.7) & 0.092 \\
\hline $\mathrm{PP}(\mathrm{mmHg})$ & 55.20 & $(17.1)$ & 69.0 & $(17.1)$ & 0.001 \\
\hline Cross sectional aortic volume $(\mathrm{mL})$ & 5.0 & $(1.9)$ & 5.1 & $(2.3)$ & 0.634 \\
\hline Aortic distensibilty $\left(\times \mathrm{IO}^{-3} \mathrm{mmHg}^{-1}\right)$ & 2.4 & $(2.0)$ & 2.1 & $(2.1)$ & 0.009 \\
\hline Aortic volumetric arterial strain & 0.12 & $(0.09)$ & 0.15 & $(0.10)$ & 0.176 \\
\hline
\end{tabular}

Results are show as mean with standard deviation in parenthesis or number with percentage in parenthesis as appropriate except for RRT time and measures of vascular function which are displayed as median and inter quartile range. Tests of significance are t-test and Chi-squared between groups, except for measures of vascular function where Mann-Whitney- $U$ was used. 


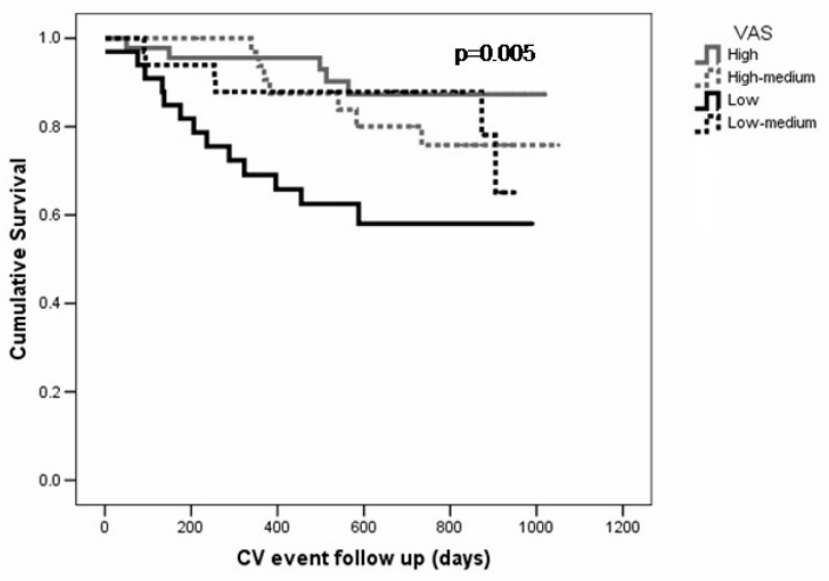

Figure 4

Kaplan-Meier survival curves for survival to either CV end point or death with patients stratified by aortic VAS quartile.

One major potential limitation of our study is that calculation of AD is based on cross-sectional volume of the vessel wall and pulse pressure. For practical reasons, direct aortic blood pressure measurement has been substituted by non-invasive indirect brachial blood pressure. Brachial blood pressure is not entirely representative of central haemodynamics and our methods do not permit assessment of the phenomenon of central to peripheral systolic and pulse pressure amplification, as can be measured with tonometry. It is difficult to dissociate any clinical effect associated with changes in $\mathrm{AD}$ from that due directly to pulse pressure, which is a well described predictor of outcome in ESRD. Nonetheless, as it is otherwise impossible to assess aortic stiffness without documentation of pressure within the vessel lumen, the approach used in this study has been used widely[7,9-11]. For this reason, aortic VAS, the fractional increase in aortic volume during the cardiac cycle was also used. This does not depend on blood pressure variables and in this study displayed similar relationships with clinical variables and outcome. However, there is limited literature to support its use. Other studies have demonstrated that PWV can be measured with CMR. There are advantages to using measuring PWV compared to AD as it is load-independent, negating the necessity for blood pressure measurements. However, at the time of initiation of this study, there were few studies in any patient group other than healthy volunteers $[10,12]$, and unfortunately CMR derived PWV was not measured in this study cohort.

The relationship between these markers of aortic function and LV dimensions show a weak but significant relationship between increased aortic stiffness and increasing LVMI and end systolic volume, suggesting that reduced AD may increase LV wall tension and hence cardiac hypertrophy. Aortic VAS, which is independent of blood pressure, correlated with markers of systolic function, namely ejection function and stroke volume, suggesting that in the failing heart where systolic blood pressure is lower, increased arterial stiffness remains deleterious to ventricular performance.

A variety of studies have addressed the role of $\mathrm{AD}$ as a marker of either CV risk or relating AD to cardiac performance in other groups at high risk of CV disease. Data relating CMR measures of arterial function to long term outcome are scarce. In otherwise healthy individuals, obese subjects have been shown by CMR to have increased aortic cross sectional area and decreased aortic elasticity $[13,14]$. AD is reduced in patients with heart failure and correlates with exercise capacity[9]. One study in hypertensive patients has demonstrated that AD increases following treatment with nicardipine or alacepril but not trichlormethiazide, independent of changes in pulse pressure[15]. More recently, in patients with non diabetic CKD, AD has been shown to correlate with GFR demonstrating that CMR can quantify the impact of reduced kidney function on vascular function in early CKD[7].

Table 3: Patient survival

\begin{tabular}{|c|c|c|c|c|c|c|}
\hline \multirow[b]{2}{*}{ Variable } & \multicolumn{3}{|c|}{ Univariate analysis } & \multicolumn{3}{|c|}{ Multivariate analysis } \\
\hline & HR & $(95.0 \% \mathrm{Cl})$ & $\mathbf{p}$ & HR & $(95.0 \% \mathrm{Cl})$ & $\mathbf{p}$ \\
\hline Diabetes & 3.031 & $(1.036,8.866)$ & 0.043 & 4.214 & $(1.631,10.886)$ & 0.003 \\
\hline $\mathrm{SBP}(\mathrm{mmHg})$ & 1.015 & $(0.992,1.038)$ & 0.197 & 1.022 & $(1.000,1.044)$ & 0.049 \\
\hline Log aortic distensibilty & 0.072 & $(0.008,0.694)$ & 0.023 & 0.135 & $(0.019,0.948)$ & 0.044 \\
\hline Log aortic VAS & 0.107 & $(0.007,1.732)$ & 0.116 & & & \\
\hline Duration RRT (days) & 1.000 & $(1.000,1.000)$ & 0.421 & & & \\
\hline Age (years) & 1.015 & $(0.969,1.063)$ & 0.526 & & & \\
\hline Gender (ref male) & 0.833 & $(0.3 \mid 2,2.226)$ & 0.716 & & & \\
\hline Haemoglobin (g/dL) & 0.910 & $(0.670,1.236)$ & 0.547 & & & \\
\hline Albumin $(g / L)$ & 0.942 & $(0.839,1.058)$ & 0.311 & & & \\
\hline
\end{tabular}

Cox survival analysis for patient survival. Because of their close correlation SBP aortic distensibilty and aortic VAS were entered separately into the model. Results for other variables are shown with systolic blood pressure in the model 
Table 4: Combined CV event and death survival

\begin{tabular}{|c|c|c|c|c|c|c|}
\hline \multirow[b]{2}{*}{ Variable } & \multicolumn{3}{|c|}{ Univariate analysis } & \multicolumn{3}{|c|}{ Multivariate analysis } \\
\hline & HR & $(95.0 \% \mathrm{CI})$ & $\mathbf{p}$ & HR & $(95.0 \% \mathrm{Cl})$ & $\mathbf{p}$ \\
\hline Diabetes & 2.989 & (1.276 7.004) & 0.012 & 3.575 & $(1.6697 .655)$ & 0.001 \\
\hline Log aortic distensibilty & 0.052 & $(0.0090 .319)$ & 0.001 & 0.066 & $(0.0130 .347)$ & 0.001 \\
\hline Log aortic VAS & 0.021 & $(0.0020 .206)$ & 0.001 & 0.026 & $(0.0040 .175)$ & $<0.001$ \\
\hline Duration RRT (days) & 1.000 & $(1.0001 .000)$ & 0.560 & & & \\
\hline $\mathrm{SBP}(\mathrm{mmHg})$ & 1.014 & (0.995 I.032) & 0.142 & & & \\
\hline Age (years) & 1.023 & $(0.984 \quad 1.062)$ & 0.248 & & & \\
\hline Gender (ref male) & 0.827 & $(0.368 \quad 1.860)$ & 0.646 & & & \\
\hline Haemoglobin (g/dL) & 0.958 & $(0.748 \quad 1.225)$ & 0.731 & & & \\
\hline Albumin $(g / L)$ & 0.984 & $(0.896 \quad \mathrm{I} .080)$ & 0.728 & & & \\
\hline
\end{tabular}

Cox survival analysis for patient survival to either death or cardiovascular event. Because of their close correlation SBP aortic distensibilty and aortic VAS were entered separately into the model. Results for other variables are shown with systolic blood pressure in the model.

Additionally, endothelial function can be studied using CMR to by assessing cross sectional flow mediated dilation of the brachial artery[16].

Independent predictors of mortality during the follow up period were diabetes, $\mathrm{AD}$ and systolic blood pressure. Due to their close interdependence, systolic blood pressure and aortic distensibilty could not be independently assessed. When a combined end point of death, non-fatal myocardial infarction, cardiac revascularisation, amputation for peripheral vascular disease and cerebrovascular event was used, only diabetes and AD or VAS were independent predictors of events. Surprisingly, age was not a predictor of outcome, suggesting that since age and AD were closely correlated, vascular, rather than temporal, aging is a more important determinant of survival. In keeping with other studies, time on renal replacement therapy was significantly longer in those patients who died[4], but this was not independently associated with outcome. Therefore in this patient group, $\mathrm{AD}$ was a predictor of mortality and/or vascular events independent of age and dialysis vintage.

Haemodynamic factors which promote arterial remodelling present in ESRD, although not specific to kidney disease, include age and blood pressure. Factors specific to ESRD include arteriovenous shunts and chronic volume overload[17]. Increased blood flow through a shunt promotes arterial remodelling although may not lead to detectible changes in measures of vascular function[18]. We could find no difference in AD or VAS in patients with an arteriovenous shunt and those without. As novel marker of increased CV risk, no data exist on the natural history of progression of $\mathrm{AD}$, or whether it can be improved by therapeutic intervention. Our results suggest that as there was no significant difference between those CKD 5 patients on dialysis compared to pre-dialysis patients, the major derangement in vascular function occurs earlier during the progression of CKD. One assumes that reduced AD is a consequence of arterial calcification[19], although we cannot confirm this as CMR is unable to display calcific lesions due to their absence of water content.

A large number of studies have been performed to assess the determinants of arterial stiffness in ESRD, and its relation to long term survival, using either incremental elastic modulus, PWV or augmentation index as markers of arterial stiffness. In keeping with our results, factors associated with increased arterial stiffness include age, diabetes, and systolic blood pressure (or pulse pressure) [20-22]. Other factors associated with arterial stiffness include serum calcium or the presence of inflammation[22]. Surprisingly, these factors were not predictors of vascular function in our study. Arterial stiffness has been repeatedly demonstrated to be an independent predictor of all cause and cardiovascular mortality in haemodialysis patients[3,4 ,23].

Aside from the issue of use of brachial blood pressure to calculate central haemodynamics, our study has some other limitations. Although direct comparison between CMR and calcification is not possible, CMR has emerged as a technique for imaging atheroma. A direct comparison between vascular function and dark blood imaging looking at aortic wall thickness and atheroma would be of interest but unfortunately was not performed. Measurement of $\mathrm{AD}$ is still a relatively novel technique and its widespread reproducibility is yet to be established. In house blinded analysis has demonstrated an interobserver variability of $4.6 \%$, although this needs to be reproduced in other centres, particularly as there are potential sources of error in both data acquisition and analysis as well as in blood pressure measurements. As we have previously used CMR with late gadolinium enhancement (LGE) to analyse myocardial tissue composition in ESRD[5], it would have been of interest to compare myocardial tissue abnormalities with vascular function. How- 
ever, as a link between gadolinium and nephrogenic systemic fibrosis has emerged, this comparison is impossible[24]. It is unfortunate that it was not possible to acquire brachial blood pressure on every patient due to some patients having multiple vascular procedures on both upper limbs. Finally, CMR technique is relatively expensive and time consuming to analyse. With future development of automated analysis software, faster analysis of $\mathrm{AD}$ will be possible.

\section{Conclusion}

This study has demonstrated that vascular function can be measured using CMR in ESRD. Increased aortic stiffness, indicated by reduced $A D$ and reduced VAS are associated with risk factors for CV disease. Both $\mathrm{AD}$ and VAS were independent predictors of combined vascular events and mortality. AD predicted all cause mortality. Therefore, CMR offers a novel non-invasive tool to assess vascular function in patients ESRD. AD and VAS are potential targets for therapeutic intervention to reduce cardiovascular risk in ESRD.

\section{Competing interests}

The authors declare that they have no competing interests.

\section{Authors' contributions}

Guarantor of integrity of entire study PBM, study concepts/study design PBM, AD, KGB, TS, JEF, HJD, AGJ data collection and analysis PBM, AD, KGB, RKP, RAPW, TS, JE, AGJ, statistical analysis PBM, RKP, AGJ, manuscript drafting or manuscript revision for important intellectual content, all authors; manuscript final version approval, all authors.

\section{Acknowledgements}

Patrick Mark was funded by a British Heart Foundation Junior Fellowship. The Glasgow Cardiac Magnetic Resonance Unit is supported by a British Heart Foundation grant. The study was additionally supported by Darlinda's Charity for Renal Research and the Chief Scientist's Office, Scotland.

\section{References}

I. Zager PG, Nikolic J, Brown RH, Campbell MA, Hunt WC, Peterson D, Van Stone J, Levey A, Meyer KB, Klag MJ, Johnson HK, Clark E, Sadler JH, Teredesai P: "U" curve association of blood pressure and mortality in hemodialysis patients. Medical Directors of Dialysis Clinic, Inc. Kidney Int 1998, 54:56I-569.

2. Port FK, Hulbert-Shearon TE, Wolfe RA, Bloembergen WE, Golper TA, Agodoa LY, Young EW: Predialysis blood pressure and mortality risk in a national sample of maintenance hemodialysis patients. Am J Kidney Dis 1999, 33:507-5I7.

3. London GM, Blacher J, Pannier B, Guerin AP, Marchais SJ, Safar ME: Arterial wave reflections and survival in end-stage renal failure. Hypertension 200I, 38:434-438.

4. Blacher J, Guerin AP, Pannier B, Marchais SJ, Safar ME, London GM: Impact of aortic stiffness on survival in end-stage renal disease. Circulation 1999, 99:2434-2439.

5. Mark PB, Johnston N, Groenning BA, Foster JE, Blyth KG, Martin TN, Steedman T, Dargie HJ, Jardine AG: Redefinition of uremic cardiomyopathy by contrast-enhanced cardiac magnetic resonance imaging. Kidney Int 2006, 69:1839-1845.

6. Zimmerli LU, Mark PB, Steedman T, Foster JE, Berg GA, Dargie HJ, Jardine $A G$, Delles $C$, Dominiczak AF: Vascular function in patients with end-stage renal disease and/or coronary artery disease: a cardiac magnetic resonance imaging study. Kidney Int 2007, 71:68-73.

7. Edwards NC, Ferro CJ, Townend JN, Steeds RP: Aortic Compliance and Arterial-Ventricular Coupling in Early Chronic Kidney Disease: a Pattern Resembling Heart Failure with Preserved Ejection Fraction. Heart 2008- in press.

8. K/DOQI clinical practice guidelines for chronic kidney disease: evaluation, classification, and stratification. Am J Kidney Dis 2002, 39:SI-266.

9. Rerkpattanapipat P, Hundley WG, Link KM, Brubaker PH, Hamilton CA, Darty SN, Morgan TM, Kitzman DW: Relation of aortic distensibility determined by magnetic resonance imaging in patients $>$ or $=60$ years of age to systolic heart failure and exercise capacity. Am J Cardiol 2002, 90:I22I-I 225.

10. Laffon E, Marthan R, Montaudon M, Latrabe V, Laurent F, Ducassou $D$ : Feasibility of aortic pulse pressure and pressure wave velocity MRI measurement in young adults. J Magn Reson Imaging 2005, $21: 53-58$.

II. Argyropoulou MI, Kiortsis DN, Daskas N, Xydis V, Mavridis A, Efremidis SC, Siamopoulou A: Distensibility and pulse wave velocity of the thoracic aorta in patients with juvenile idiopathic arthritis: an MRI study. Clin Exp Rheumatol 2003, 21 :794-797.

12. Rogers WJ, Hu YL, Coast D, Vido DA, Kramer CM, Pyeritz RE, Reichek N: Age-associated changes in regional aortic pulse wave velocity. J Am Coll Cardiol 200I, 38: I I23-I I 29.

13. Danias PG, Tritos NA, Stuber M, Botnar RM, Kissinger KV, Manning WJ: Comparison of aortic elasticity determined by cardiovascular magnetic resonance imaging in obese versus lean adults. Am J Cardiol 2003, 91 : 195-199.

14. Robinson MR, Scheuermann-Freestone M, Leeson P, Channon KM, Clarke K, Neubauer S, Wiesmann F: Uncomplicated obesity is associated with abnormal aortic function assessed by cardiovascular magnetic resonance. J Cardiovasc Magn Reson 2008, 10:10.

15. Honda T, Hamada M, Shigematsu Y, Matsumoto Y, Matsuoka H, Hiwada K: Effect of antihypertensive therapy on aortic distensibility in patients with essential hypertension: comparison with trichlormethiazide, nicardipine and alacepril. Cardiovasc Drugs Ther 1999, 13:339-346.

16. Wiesmann F, Petersen SE, Leeson PM, Francis JM, Robson MD, Wang $\mathrm{Q}$, Choudhury R, Channon KM, Neubauer S: Global impairment of brachial, carotid, and aortic vascular function in young smokers: direct quantification by high-resolution magnetic resonance imaging. J Am Coll Cardiol 2004, 44:2056-2064.

17. London GM, Guerin AP, Marchais SJ, Pannier B, Safar ME, Day M, Metivier F: Cardiac and arterial interactions in end-stage renal disease. Kidney Int 1996, 50:600-608.

18. Savage MT, Ferro CJ, Sassano A, Tomson CR: The impact of arteriovenous fistula formation on central hemodynamic pressures in chronic renal failure patients: a prospective study. Am J Kidney Dis 2002, 40:753-759.

19. London GM, Guerin AP, Marchais SJ, Metivier F, Pannier B, Adda H: Arterial media calcification in end-stage renal disease: impact on all-cause and cardiovascular mortality. Nephrol Dial Transplant 2003, 18:1731-1740.

20. Blacher J, London GM, Safar ME, Mourad JJ: Influence of age and end-stage renal disease on the stiffness of carotid wall material in hypertension. J Hypertens 1999, 17:237-244.

21. Covic A, Goldsmith DJ, Panaghiu L, Covic M, Sedor J: Analysis of the effect of hemodialysis on peripheral and central arterial pressure waveforms. Kidney Int 2000, 57:2634-2643.

22. Seyrek N, Balal M, Karayaylali I, Paydas S, Aikimbaev K, Cetiner S, Seydaoglu G: Which parameter is more influential on the development of arteriosclerosis in hemodialysis patients? Ren Fail 2003, 25: $1011-1018$.

23. Safar ME, Blacher J, Pannier B, Guerin AP, Marchais SJ, Guyonvarc'h PM, London GM: Central pulse pressure and mortality in endstage renal disease. Hypertension 2002, 39:735-738.

24. Collidge TA, Thomson PC, Mark PB, Traynor JP, Jardine AG, Morris ST, Simpson K, Roditi GH: Gadolinium-enhanced MR imaging and nephrogenic systemic fibrosis: retrospective study of a renal replacement therapy cohort. Radiology 2007, 245: 168-I75. 